\title{
COMPACT MULTIPLIERS ON BANACH ALGEBRAS
}

\author{
STEPHEN H. FRIEDBERG
}

\begin{abstract}
An elementary proof is provided to show that for a large class of
\end{abstract} Banach algebras, the compact multipliers are trivial.

In [1] Dutta and Tewari prove the above result for Segal algebras on noncompact lca groups (i.e., those lca groups with nondiscrete dual groups). We give a much simpler proof in a more general setting.

Let $A$ be a commutative semisimple Banach algebra considered as a subalgebra of $C(\Delta)$ where $\Delta$ denotes the maximal ideal space of $A$. We assume the following regularity condition: For each $x$ in $\Delta$ and neighborhood $V$ of $x$, there exists $f$ in $A$ with $\|f\| \equiv\|f\|_{A} \leqslant 1$ (or any constant independent of $x$ and $V), f(x)=1$, and whose support supp $f \subset V$.

THEOREM. Let $A$ be a Banach algebra satisfying the above conditions and let $T$ be a compact multiplier on $A$. Then $T$ is trivial if $\Delta$ contains no isolated points.

Proof. Since $T$ is a multiplier there exists a continuous function $\varphi$ on $\Delta$ such that $T(f)=\varphi f$ for all $f$ in $A$. We must show that $\varphi=0$. Suppose that there exists $x_{0}$ in $\Delta$ such that $\varphi\left(x_{0}\right) \neq 0$. Choose $a>0$ and a neighborhood $V$ of $x_{0}$ such that $|\varphi(x)| \geqslant a$ for all $x$ in $V$. Since $x_{0}$ is not an isolated point, we may choose a sequence $\left\{x_{n}\right\}$ in $V$ with disjoint neighborhoods $\left\{V_{n}\right\}$ such that $x_{n} \in V_{n} \subset V$ for all $n$. For each $n$ choose $f_{n}$ in $A$ with $f_{n}\left(x_{n}\right)=1,\left\|f_{n}\right\|<1$, and supp $f \subset V_{n}$. Then for all $n$

$$
\|T\| \geqslant\left\|\varphi f_{n}\right\| \geqslant\left\|\varphi f_{n}\right\|_{\infty} \geqslant a .
$$

Since $T$ is compact we have (passing to a subsequence) that $\varphi f_{n} \rightarrow h$ in $A$. So $h(x)=\lim \left(\varphi f_{n}\right)(x)=0$ for all $x$. But by (1) $\|h\| \geqslant a$, which is a contradiction.

\section{REFERENCES}

1. M. Dutta and B. Tewari, On multipliers of Segal algebras, Proc. Amer. Math. Soc. 72 (1978), 121-124.

Department of Mathematics, IllinOIs State University, NoRmal, Illinois 61761

Received by the editors December 7, 1978.

AMS (MOS) subject classifications (1970). Primary 43A25; Secondary 46J10.

Key words and phrases. Multiplier, Segal algebra, compact operator, semisimple. 